\title{
Automatic Identification and Elimination of Pectoral Muscle in Digital Mammograms
}

\author{
K.Vaidehi \\ Research Scholar \\ Department of Computer Science \& Technology, \\ Annamalai University \\ Annamalai nagar - 608002 , \\ T.N., India.
}

\author{
T.S.Subashini, Ph.D \\ Associate Professor \\ Department of Computer Science \& Technology, \\ Annamalai University \\ Annamalai nagar - 608002 , \\ T.N., India.
}

\begin{abstract}
Computer aided detection/diagnosis aims at assisting radiologist in the analysis of digital mammograms. Digital mammogram has emerged as the most popular screening technique for early detection of breast cancer and other abnormalities in human breast tissue. The pectoral muscle represents a predominant density region in most mammograms and can affect/bias the results of image processing methods. This paper addresses the problem of eliminating the pectoral muscles from the mammogram so that further processing for detection and diagnosis of breast cancer is confined to the breast region alone. The proposed work is done in three steps. In the first step, the mammogram is oriented to the left to minimize computations. In the second step the top left quadrant of the mammogram which contains the pectoral muscle is extracted. Next, the pectoral muscle contour is computed using our proposed algorithm. Totally 120 mammogram images were taken up for the study. A comprehensive comparison with manually-drawn contours by the radiologist reveals the strength of the proposed method and shows that it can be effectively used as a preprocessing step in the design of CAD system for breast cancer.
\end{abstract}

Keywords: Pectoral Muscle, Digital Mammograms, CAD

\section{INTRODUCTION}

In the US, breast cancer is the most common cancer amongst women and 1 in 8 women in the US have a chance of developing breast cancer in their life time. In the year 2008, there were about $1,82,000$ breast cancer cases reported in the US, whereas in India, 1,15,000 new cases were diagnosed. This implies that, though, because of India's population, the percentage of total women affected seems less, the breast cancer burden in India has almost reached about $2 / 3$ rds of that of the US and is steadily rising [1]. India is experiencing an unprecedented rise in the number of breast cancer cases across all sections of society, as are also other countries. There is no way we can prevent breast cancer, but we can definitely detect it early and treat adequately. Achieving this will lead to better long term survival as well as a better quality of life. According to Indian council of medical research statistics, 10,000 breast cancers are being diagnosed every year in India and more than $70 \%$ of them are diagnosed in advanced stage [2]. By 2020, the incidence of breast cancer in India is expected to double. The cancer registries' data shows that urban women are at almost having double the risk of breast cancer than rural women [3]. The Indian Cancer Society as declared 2013 as a breast cancer awareness year and is taking various initiatives to create awareness in people.

\subsection{Breast Cancer and Mammograms}

A mammogram is an X-ray image of the breast and it is used to aid in the early detection and diagnosis of breast diseases in men and women. Currently it is recommended that women above 40 years have to regular undergo mammogram check every year for early detection of breast cancer. It can also be used to detect and diagnose breast disease in women experiencing symptoms such as a lump, pain or nipple discharge. A digital mammogram is shown in Fig. 1.

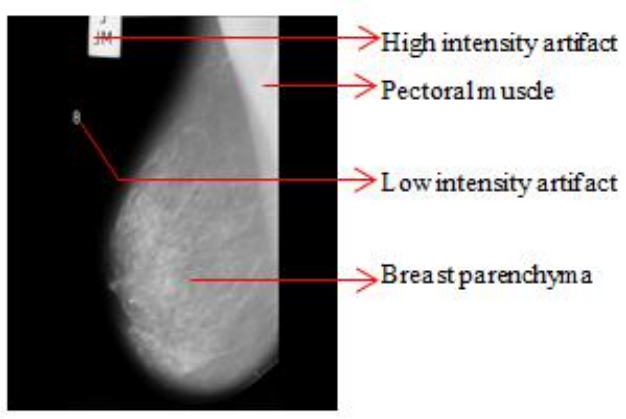

Fig.1. Digital Mammogram

\subsection{Pectoral Muscle and Need for its Elimination}

The pectoral muscle is a thick, fan-shaped muscle, situated at the upper front (anterior) of the chest wall. It makes up the bulk of the chest muscles in the male and lies under the breast in the female.

The pectoral muscle appears as a triangular opacity across the upper posterior margin of the image [4] as shown in Fig. 1. The pectoral muscle in medio-lateral oblique (MLO) mammogram images is one of the high intensity landmarks in the breast. As it can bias and affect the results of any mammogram processing method, it is often necessary to automatically identify and segment the pectoral muscle prior to breast tissue image analysis. The wide variability in the position of the muscle contour, together with the similarity between in muscle and breast tissues makes this a difficult task. 


\section{LITERATURE REVIEW}

In the literature, various methods for automatic pectoral muscle segmentation have been proposed. Pectoral boundary has been approximated by straight line in $[5,6]$. The work in [7] uses the Hough transform to identify the pectoral muscle as a straight line edge in the mammogram. Watershed transform to the gradient of an image and merging algorithm was proposed in [8] to fuse the over segmented pectoral muscle region to acquire the pectoral muscle boundary. Seeded region growing algorithmwas implemented for pectoral muscle removal in [9]. Multi resolution technique using Gabor wavelets was proposed for identification of the pectoral muscle in [10].

Detection of pectoral muscle boundary based on discrete Markov chain model (DTMC) and active contour model was proposed in [11], while the work in [12] detected the pectoral muscle contour using support vector regression models. Rough border of the pectoral muscle was found by combining the iterative Otsu thresholding and the mathematical morphological processing in [13]. Here the multiple regression analysis (MRA) is employed to obtain the pectoral muscle. In our previous work histogram-based thresholding technique and connected component labeling is employed for pectoral muscle suppression [14]. An active contours and a stopping algorithm was employed in [15] to find the pectoral muscle boundary.

\section{METHODOLOGY AND RESULTS}

As an initial step the high intensity artifacts present in the image is removed by applying connected component labeling. The biggest component which contains the breast profile including the pectoral muscle is retained and the remaining components are removed as shown in Fig. 2.

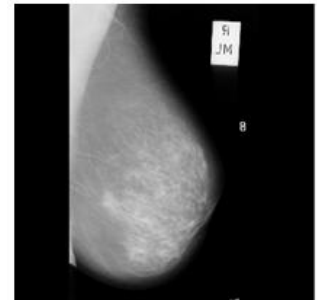

(a)

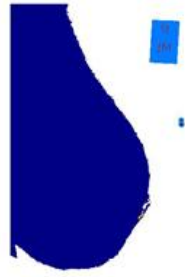

(b)

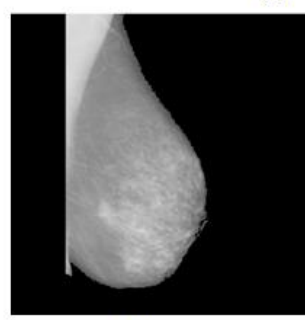

(c)

Fig. 2 a) Original Image. b) Connected component labeled image. c) Artifacts removed image.
The steps proposed to remove the pectoral region are as follows:

1. $1024 \times 1024$ size artifact less MLO mammogram is taken as input as shown in Fig. 3a.

2. To make processing easy the right MLO mammogram is flipped before removing the pectoral region.

3. The given MLO mammogram image is partitioned into four quadrants of $512 \times 512$ each. The top left quadrant $512 \times 512$, which contains the pectoral muscle is the region of interest and at the pixels of the other three quadrants are changed to black as shown in Fig. $3 b$ and Fig. 3c.

4. The top left quadrant given in Fig. 3c. is processed further to detect the pectoral muscle and the steps involved are as follows:

i. The upper quadrant is divided into 4 parts of size 128 X 512 as shown in Fig.4a.

ii. Then pixel at location $(128,512)$ is taken as point $\mathrm{A}$ and the non-zero pixel in the last row $\left(512^{\text {th }}\right.$ row $)$ is taken as point $\mathrm{B}$ as shown in Fig. $4 \mathrm{~b}$.

iii. The region above line $\mathrm{AB}$ which contains the pectoral region (Fig. 4c.) is now thresholded with the value of 176 to obtain the binary image as shown in Fig. 4d. The threshold value of 176 is found out experimentally as the pectoral region appears as high intensity regions in the mammogram.

5. The binary image is compared with the original image and the pectoral muscle is removed by replacing the original image with intensity value of zero in the locations where the intensity is 255 in the binary image. The results are shown in Fig. 5a, Fig. 5b and Fig. 5c.

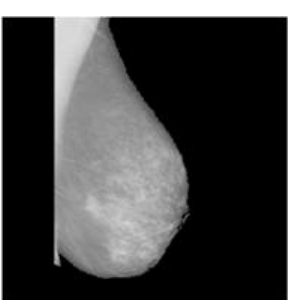

(a)

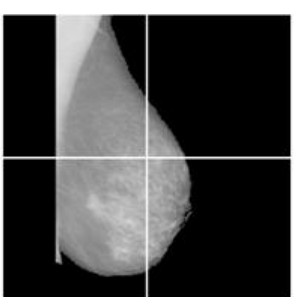

(b)

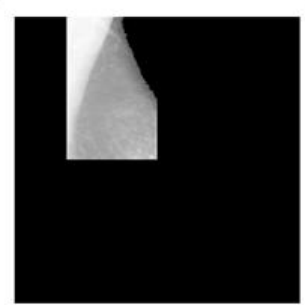

(c)

Fig.3 a) 1024 x 1024 Input image. b) Four quadrants. c) Left top quadrant (ROI) and other three quadrants changed to black. 


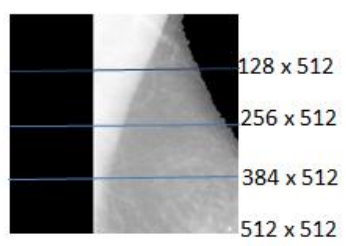

(a)

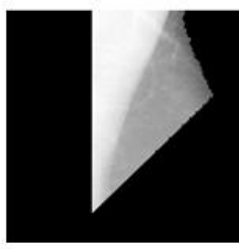

(c)

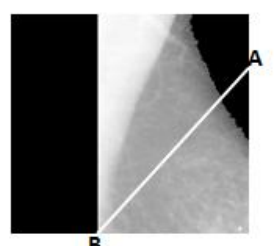

(b)

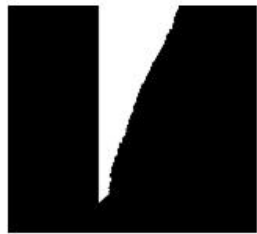

(d)
Fig. 4 Pectoral muscle segmentation.a) Top left quadrant divided into 4 parts. b) Points $A$ and $B$ identified. c) Region above the line AB. d) Segmented pectoral muscle region.

In totality 120 mammogram images from the Mini-Mias database were taken up for the study and this work has been implemented in MATLAB. The results obtained were compared with manually drawn contours by the radiologist and the accuracy obtained was $85 \%$. Out of 120 images 11 images got over segmented and 7 images got under segmented and the true positive rate achieved was $90.2 \%$. Visual inspection of the results reveals that there was over segmentation of the breast in case with dense tissue. Table 1 shows the comparison between proposed work and previous works.

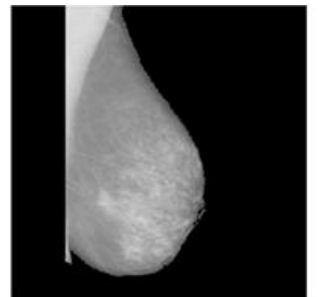

(a)

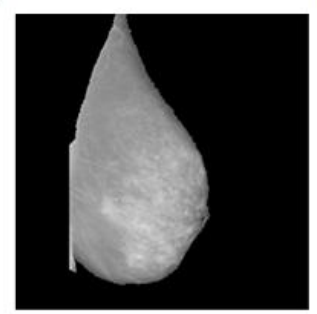

(c)

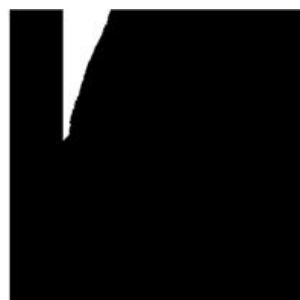

(b)
Fig. 5 a) Original image. b) Segmented pectoral muscle region. c) Pectoral muscle eliminated breast region.
Table 1. Comparison between proposed work and previous works

\begin{tabular}{|l|l|l|l|l|}
\hline S.No. & \multicolumn{1}{|c|}{ Method } & Accuracy & \multicolumn{1}{|c|}{ Reference } & Year \\
\hline 1. & $\begin{array}{l}\text { Mean shift } \\
\text { segmentation }\end{array}$ & $84 \%$ & $\begin{array}{l}\text { Sultana et al., } \\
{[17]}\end{array}$ & 2010 \\
\hline 2. & $\begin{array}{l}\text { Discrete time } \\
\text { Markov chain }\end{array}$ & $84 \%$ & $\begin{array}{l}\text { Wang et al., } \\
{[11]}\end{array}$ & 2010 \\
\hline 3. & AD method & $81 \%$ & Liu et al., [16] & 2011 \\
\hline 4. & Straight line & $85 \%$ & proposed & 2013 \\
\hline
\end{tabular}

\section{CONCLUSION}

A new method for pectoral muscle detection and elimination is proposed in this paper. Totally 120 mammogram images were taken up for the study. The accuracy obtained was $85 \%$ and the true positive rate achieved was $90.2 \%$. There was over segmentation in case of breast with dense parenchyma. This is justified because there is only a slight intensity variation between dense breast tissue and pectoral muscle and the pectoral boundary is ill defined in the case of dense breast. A comprehensive comparison with manually-drawn contours by the radiologist reveals the strength of the proposed method. The results further show that the proposed method can be effectively used as a preprocessing step in the design of CAD system for breast cancer.

\section{ACKNOWLEDGEMENT}

The authors would like to thank Dr.M.K.Sivakkolunthu, Professor of Radiology, Raja Muthiah Medical College Hospital, Annamalai Nagar for his valuable help and comments in carrying out this work.

\section{REFERENCES}

[1] Consolidated Report of the PBCR's 2006-2008, NCRP (National Cancer Registry Program), www.breastcancerindia.net/bc/statistics/stat_global.htm

[2] Vaidehi, K., and T. S. Subashini. "A global approach for detecting mass in Digital Mammograms." International Journal of Advancements in Research \& Technology, (2012).

[3] ABSICON 2012, The First International Congress of the Association of Breast Surgeons of India, (2012). www.raagalahari.com

[4] Eklund, G. W., Gilda Cardenosa, and Ward Parsons. "Assessing adequacy of mammographic image quality." Radiology 190, no. 2 (1994): 297-307.

[5] Georgsson, F. "A transformation of mammograms based on anatomical features: in digital mammography." In Proceedings of the 5th International Workshop on Digital Mammography, Madison, WI, Medical Physics Publishing, pp. 721-726. (2001). 
[6] Kwok, S. M., R. Chandrasekhar, and Y. Attikiouzel. "Automatic pectoral muscle segmentation on mammograms by straight line estimation and cliff detection." In Intelligent Information Systems Conference, The Seventh Australian and New Zealand 2001, pp. 67-72. IEEE, (2001).

[7] Karssemeijer, Nico. "Automated classification of parenchymal patterns in mammograms." Physics in medicine and biology 43, no. 2 (1998): 365.

[8] Camilus, K. Santle, V. K. Govindan, and P. S. Sathidevi. "Pectoral muscle identification in mammograms." Journal of Applied Clinical Medical Physics 12, no. 3 (2011).

[9] Nagi, Jawad, S. Abdul Kareem, FarrukhNagi, and S. Khaleel Ahmed. "Automated breast profile segmentation for ROI detection using digital mammograms." In Biomedical Engineering and Sciences (IECBES), 2010 IEEE EMBS Conference on, pp. 87-92. IEEE, (2010).

[10] Ferrari, Ricardo J., Rangaraj M. Rangayyan, JE Leo Desautels, R. A. Borges, and Annie France Frere. "Automatic identification of the pectoral muscle in mammograms." Medical Imaging, IEEE Transactions on 23, no. 2 (2004): 232-245.

[11] Wang, Lei, Miao-liang Zhu, Li-ping Deng, and Xin Yuan. "Automatic pectoral muscle boundary detection in mammograms based on markov chain and active contour model." Journal of Zhejiang University SCIENCE C 11, no. 2 (2010): 111-118

[12] Domingues, I., J. S. Cardoso, I. Amaral, I. Moreira, P. Passarinho, J. Santa Comba, R. Correia, and M. J.
Cardoso. "Pectoral muscle detection in mammograms based on the shortest path with endpoints learnt by SVMs." InEngineering in Medicine and Biology Society (EMBC), 2010 Annual International Conference of the IEEE, pp. 3158-3161. IEEE, (2010).

[13] Liu, Chen-Chung, Chung-Yen Tsai, Jui Liu, Chun-Yuan $\mathrm{Yu}$, and Shyr-Shen Yu. "A pectoral muscle segmentation algorithm for digital mammograms using Otsu thresholding and multiple regression analysis." Computers \& Mathematics with Applications 64, no. 5 (2012): 1100-1107.

[14] Subashini, T. S., VennilaRamalingam, and S. Palanivel. "Automated assessment of breast tissue density in digital mammograms." Computer Vision and Image Understanding 114, no. 1 (2010): 33-43.

[15] Akram, F., Kim, J.H., Whoang,I., and Choi, K.N., “ A preprocessing algorithm for the CAD system of mammograms using the active contour method." Applied Medical Informatics, 32(2) (2013) : 1-13.

[16] Liu, Li, Jian Wang, and Tianhui Wang. "Breast and pectoral muscle contours detection based on goodness of fit measure." In Bioinformatics and Biomedical Engineering,(iCBBE) 2011 5th International Conference on, pp. 1-4. IEEE, 2011.

[17] Sultana, Alina, Mihai Ciuc, and Rodica Strungaru. "Detection of pectoral muscle in mammograms using a mean-shift segmentation approach." InCommunications (COMM), 2010 8th International Conference on, pp. 165-168. IEEE, 2010. 